\title{
Influence of weeks of circadian misalignment on leptin levels
}

This article was published in the following Dove Press journal:

Nature and Science of Sleep

16 December 2009

Number of times this article has been viewed

\section{June Nguyen \\ Kenneth PWright Jr}

Department of Integrative Physiology, Sleep and Chronobiology Laboratory, University of Colorado, Boulder, CO, USA
Correspondence: Kenneth Wright University of Colorado, Department of Integrative Physiology, Sleep and Chronobiology Laboratory, 1725 Pleasant Street; Clare Small II 4, Boulder. CO 80309-0354, USA

Tel + I 303735 | 923

Fax +I 3034924009

Email kenneth.wright@colorado.edu
Abstract: The neurobiology of circadian, wakefulness-sleep, and feeding systems interact to influence energy homeostasis. Sleep and circadian disruptions are reported to be associated with increased risk of diabetes and obesity, yet the roles of energy balance hormones in these associations are largely unknown. Therefore, in the current study we aimed to assess the influence of several weeks of circadian misalignment (sleep and wakefulness occurring at an inappropriate biological time) on the anorexigenic adipocyte hormone leptin. We utilized data from a previous study designed to assess physiological and cognitive consequences of changes in day length and light exposure as may occur during space flight, including exploration class space missions and exposure to the Martian Sol (day length) We hypothesized that circadian misalignment during an exploration class spaceflight simulation would reduce leptin levels. Following a three-week $\sim 8$ hours per night home sleep schedule, 14 healthy participants lived in the laboratory for more than one month. After baseline data collection, participants were scheduled to either 24.0 or 24.6 hours of wakefulness-sleep schedules for 25 days. Changes in the phase of the circadian melatonin rhythm, sleep, and leptin levels were assessed. Half of participants analyzed exhibited circadian misalignment with an average change in phase angle from baseline of $\sim 4$ hours and these participants showed reduced leptin levels, sleep latency, stage 2 and total sleep time ( 7.3 to 6.6 hours) and increased wakefulness after sleep onset (all $P<0.05$ ). The control group remained synchronized and showed significant increases in sleep latency and leptin levels. Our findings indicate that weeks of circadian misalignment, such as that which occurs in circadian sleep disorders, alters leptin levels and therefore may have implications for appetite and energy balance.

Keywords: circadian disruption, circadian entrainment, circadian misalignment, sleep, sleep loss, leptin

\section{Introduction}

Leptin is an anorexigenic hormone, which acts on the hypothalamus to influence energy balance, metabolism, and feeding behaviors. Circulating leptin levels are reported to represent total body lipid stores and to be involved in long term energy balance. ${ }^{1}$ Leptin levels are also reported to show a diurnal variation. In general, leptin levels are lowest in the early morning, increase across the day and are highest during sleep at night. ${ }^{2,3}$ This diurnal leptin profile has been observed regardless of standard meal intake or continuous enteral nutrition. ${ }^{4-8}$ Factors that are reported to influence 24-hour leptin levels include food restriction and overfeeding, ${ }^{9,10}$ sleep loss and sleep extension $^{6,11,12}$ and circadian phase..$^{5,13}$ 
For example, in humans, caloric restriction is reported to decrease leptin levels, whereas caloric excess is reported to increase leptin levels..$^{9,10,14}$ Elimam and Marcus ${ }^{10}$ reported that two days of fasting reduced leptin levels by $60 \%$ and that under such fasting conditions leptin levels showed no diurnal variation. Chin-Chance and colleagues ${ }^{9}$ reported that three days of food restriction to $\sim 70 \%$ of energy requirements reduced leptin levels on average by $26 \%$ and that three days of overfeeding by $\sim 130 \%$ increased leptin levels by $\sim 21 \%$. With respect to sleep, Schoeller and colleagues ${ }^{2}$ and Simon and colleagues ${ }^{5}$ reported higher leptin levels during a daytime sleep episode as compared to levels during a daytime wakefulness episode, suggesting that sleep may increase leptin levels. Taheri and colleagues ${ }^{15}$ examined participants in the Wisconsin Sleep Cohort Study and reported that short sleep duration was significantly associated with low morning leptin levels; independent of age, body mass index (BMI), and gender. Spiegel and colleagues ${ }^{12}$ reported a $19 \%$ reduction in 24-hour leptin levels following six days sleep restriction verses sleep extension. Spiegel and colleagues ${ }^{11}$ also reported that two days of sleep restriction versus sleep extension reduced daytime leptin levels by $18 \%$. Furthermore, the amplitude of the 24-hour leptin profile has been reported to be reduced during 88 hours of total sleep deprivation. ${ }^{6}$

The internal circadian timekeeping system may also modulate leptin levels. A master circadian clock that regulates near 24-hour rhythms in mammalian physiology is located in the hypothalamic suprachiasmatic nucleus (SCN). ${ }^{16}$ The circadian rhythm of the pineal hormone melatonin is the most commonly used endocrine marker representing the output of the circadian clock in humans. Low melatonin levels represent the biological day and high melatonin levels represent the biological night. During synchronization to the 24-hour day (ie, entrainment), the phase of the melatonin rhythm occurs at a similar time each day relative to the wakefulnesssleep schedule and environmental time. ${ }^{17}$ The circadian timekeeping system promotes wakefulness and its associated functions (eg, activity, nutrition intake and metabolism) during the solar day and promotes sleep and its associated functions (eg, rest, growth, and protein synthesis) during solar darkness. Sleep is disturbed at a biological time when endogenous melatonin levels are low. ${ }^{8,18,19}$ Nonsynchronization (ie, misalignment) between sleep-wakefulness schedules and internal biological time is common in today's 24-hour society because of occupations that require shift work, ${ }^{20}$ frequent air travel across many time zones,${ }^{21}$ and during space flight and submarine missions. ${ }^{18,22}$ Circadian misalignment is also common in circadian rhythm sleep disorders ${ }^{23}$ and in psychiatric disorders. ${ }^{24}$ Such altered work schedules and disorders result in people being awake during the biological night when levels of the sleep-promoting hormone melatonin are high. This disruption in circadian physiology can lead to functional and clinical impairments. ${ }^{25,26}$ With respect to circadian misalignment, Simon and colleagues ${ }^{5}$ reported that leptin levels increased at night and decreased in the morning regardless of whether participants were asleep or awake suggesting that the circadian system influences leptin levels. Sympathetic projections from the SCN to the paraventricular nucleus to adipose tissue ${ }^{27}$ provide a possible mechanism for circadian regulation of leptin. Using a constant routine protocol, Shea and colleagues ${ }^{13}$ reported leptin levels to peak in the early morning hours and that the circadian amplitude of the leptin rhythm was approximately $10 \%$ of the overall diurnal profile. Using a forced desynchrony protocol, Scheer and colleagues ${ }^{8}$ reported leptin levels were acutely responsive to being awake at the wrong internal biological time such that leptin levels were lower when sleep occurred during the biological day.

The findings reviewed above suggest that sleep loss and changes in circadian time may alter the amplitude and shape of the 24-hour leptin profile. ${ }^{13}$ Changes in the 24-hour leptin profile may influence food intake and energy balance. Such changes in leptin may contribute to the increased risk of obesity, which is associated with sleep loss ${ }^{10}$ and shift work ${ }^{28}$ by altering energy balance via changes in food intake. It is unknown how energy balance hormones such as leptin are affected by weeks of misalignment between internal biological time, sleep and wakefulness schedules. The latter question was therefore examined in the current study, which was designed as a ground based model of an exploration classed space mission. ${ }^{19,29}$ Specifically, we hypothesized that a failure to maintain synchronization between the internal circadian clock and the wakefulness-sleep schedule would reduce circulating leptin levels.

\section{Participants and methods Participants}

Fourteen healthy participants from the Boston area (12 males and 2 females aged $31.6 \pm 5.9$ years [mean $\pm \mathrm{SD}$ ]) with available blood samples from previously published studies ${ }^{19,29}$ were included in the current analyses. Average body mass index was $24.3 \pm 2.3$ (mean $\pm \mathrm{SD}$ ). Seventeen participants were initially studied, however, baseline blood samples were not available for two participants and one subject's leptin levels were more than three standard deviations above the group mean and thus were not included in the analysis. 
Participants were healthy as determined by a structured interview with a clinical psychologist, medical history, physical examination, 12-lead electrocardiogram, blood and urine chemistries, and drug toxicology. Participants were nonsmokers, medication free and were instructed to refrain from caffeine, nicotine, alcohol and nonsteroidal anti-inflammatory drugs for three weeks prior to study. Participants reported no shift work within three years, or traveling across more than one time zone within three months prior to study. The study was approved by Institutional Human Subject Review Boards, and written informed consent was obtained from all the participants. The experiment was conducted in accordance with the principles in the Declaration of Helsinki.

\section{Outpatient study protocol}

The influence of prior sleep loss on leptin levels was minimized by requiring participants to maintain a regular daily routine of $\sim 8$ hours of scheduled sleep and $\sim 16$ hours of scheduled wakefulness for three weeks prior to the inpatient laboratory protocol. Sleep $\operatorname{logs}$ and call-ins into a timestamped voice recorder verified this schedule. In addition, sleep-wake schedules were verified using wrist actigraphy and ambulatory light exposure recordings (Actiwatch-L, Mini Mitter, Bend, OR) at least one week before laboratory admission. The participants' self-selected 16:8 hour wakefulnesssleep schedule during the week prior to laboratory admission was used to calculate their habitual bedtime. Habitual bedtime was determined by subtracting four hours from the average midpoint time of the $\sim 8$-hour sleep schedule.

\section{Inpatient study protocol}

Participants were studied in an environment free of time cues in individual rooms located on a General Clinical Research Center (GCRC). During the first six days, participants were scheduled to a 16-hour wakefulness-8-hour sleep cycle with sleep scheduled at habitual bedtime. Blood was collected on day 6 to assess 24-hour baseline leptin levels. Blood sampling began at scheduled waketime for 12 participants and at scheduled bedtime for two participants, the later because of blood sampling difficulty. Following the six baseline days, participants underwent a constant routine protocol to estimate circadian phase. ${ }^{30,31}$ Participants were then scheduled to either a 24.0-hour or 24.6-hour day for up to 25 days; described in greater detail previously. ${ }^{19,29}$ On day $\sim 22$ of the protocol, following more than two weeks on the 24.0-hour or 24.6-hour schedules, blood was sampled to reassess 24-hour leptin levels (Figure 1). For eight participants, blood was not available on day 20 and thus available samples from one subject were used from days 21 and 30 and for two participants on day 22 and 32 of the protocol. The average day of leptin reassessment was day 22 . The average day of reassessment of the phase of the melatonin rhythm was day 23 .

Protocol events were scheduled relative to the participants' scheduled waketime, which was assigned a relative clock hour value of 0800 hours. For example, breakfast at 1 hour 35 min after scheduled waketime, would be reported as a relative clock hour of 0935 hours, regardless of the actual clock hour. Ambient light, room temperature, sleep-wake opportunities and activity were controlled. Nutrition intake (breakfast, lunch, dinner and a snack; $150 \mathrm{mEq} \mathrm{Na}{ }^{+}, 100 \mathrm{mEq}$ $\mathrm{K}^{+} \pm 20 \%, 1500$ to $2500 \mathrm{~mL}$ fluids, isocaloric) was provided at relative clock hours 0935 hours, 1300 hours, 1900 hours, and 2233 hours. The participants' weight was kept constant across the protocol by GCRC nutritionists adjusting subject's caloric intake. Exercise beyond light stretching, and napping were proscribed.

Ceiling-mounted fluorescent lamps (Phillips [Eindhoven, The Netherlands] T8 and T80) with a $4100 \mathrm{~K}$ color temperature produced a spectrum of white light. ${ }^{32}$ Lux levels were measured with an IL-1400 photometer (International Light, Inc. Newburyport, MA). Ambient light intensity was measured with the light sensor placed on a tabletop $\sim 76-\mathrm{cm}$ tall and maximum light intensity was measured at $\sim 183 \mathrm{~cm}$ with the sensor aimed in the direction of the light fixtures; angle of gaze was measured near the eye with the sensor in the direction of gaze. Lighting levels for day 1 during scheduled wakefulness were $\sim 3$ lux in the angle of gaze ( $<5$ lux ambient; $<15$ lux maximum). On days 2-6, participants were exposed to bright indoor light $(\sim 450$ lux in the angle of gaze; $<1,100$ lux ambient; $<1,500$ lux maximum) to ensure stable entrainment to the 24-hour day prior to the subsequent portions of the protocol. On day 7 during the constant routine, participants were exposed to light levels of $\sim 1.5$ lux in the angle of gaze $(<3$ lux ambient at $\sim 76 \mathrm{~cm},<8$ lux maximum) to accurately assess circadian melatonin phase in dim light. Hours of light and darkness were 16:8 hours for the 24.0-hour day and 16.4:8.2 hours for the 24.6-hour day. Light levels during these days were $\sim 1.5$ lux in the angle of gaze for 10 participants simulating dim light exposure on some space shuttle missions ${ }^{18}$ and were $\sim 25$ lux in the angle of gaze ( $<50$ lux ambient at $\sim 76 \mathrm{~cm},<120$ maximum) for the remaining four participants to test if brighter light was sufficient to entrain the circadian clock to the imposed day lengths. The nonsynchronized group consisted of one subject in the $\sim 25$ lux condition and six participants in the $\sim 1.5$ lux condition. 


\section{A 1839 \\ Day length $(\mathrm{T}-\mathrm{Cycle})=\mathbf{2 4 . 0 - \mathrm { h }}$ \\ Relative clock time (h)}

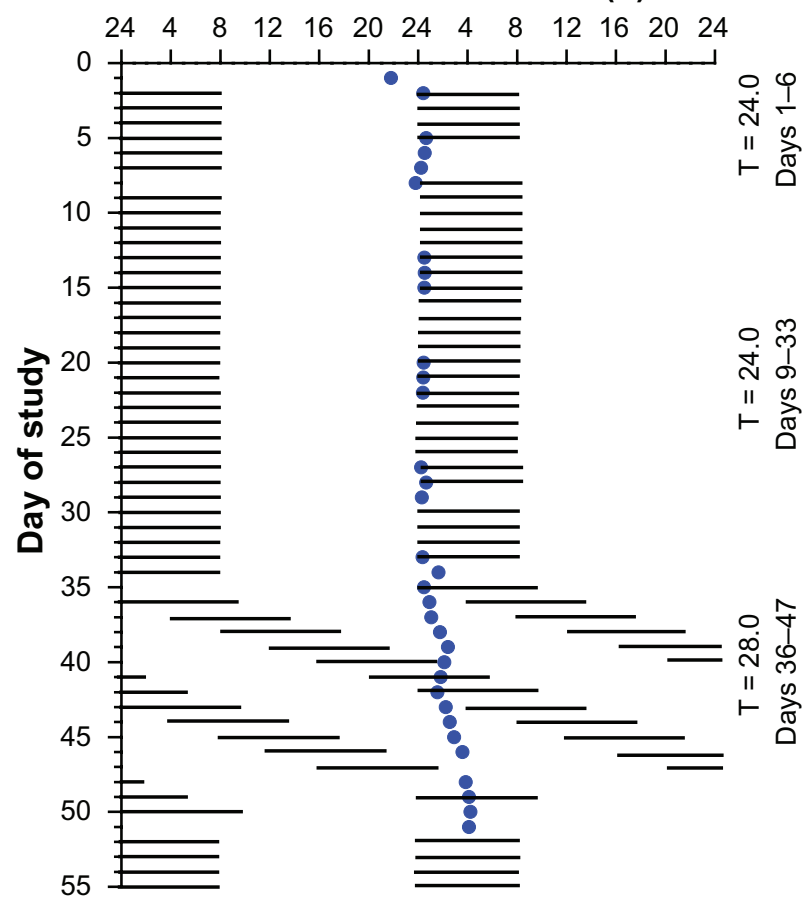

\section{B \\ $18 \mathrm{~J} 5$ \\ Day length $(\mathrm{T}-$-Cycle $)=24.6 \mathrm{~h}$ \\ Relative clock time (h)}

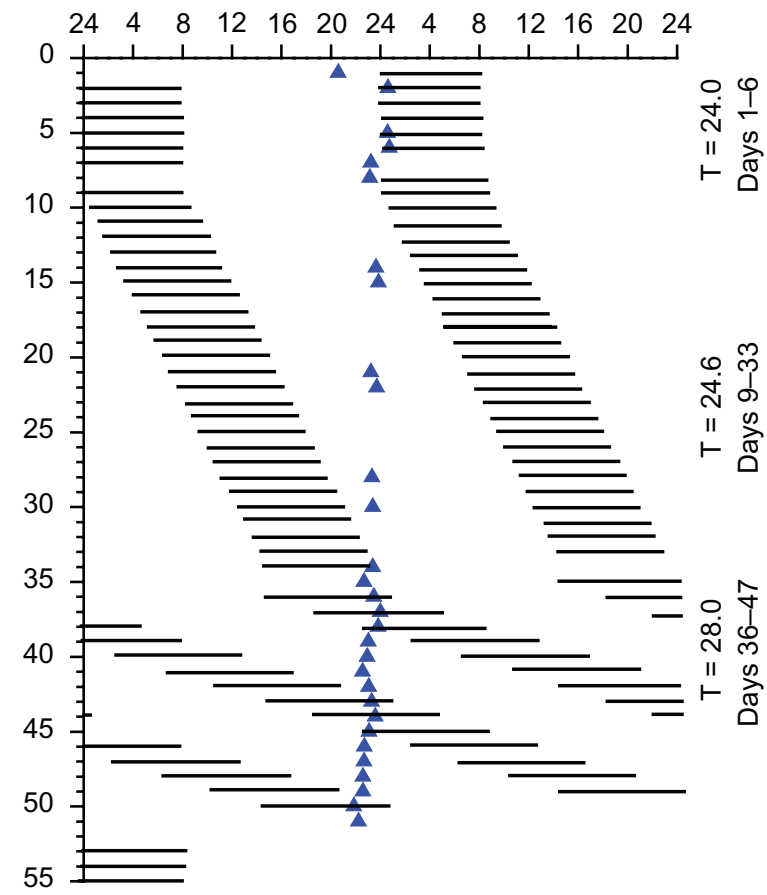

Figure I Rasterplot of the study design for two representative individuals. Rasterplot is double plotted such that consecutive days are next to and beneath the other. Data are plotted to a relative clock time with lights-out assigned a value of 24.00 hours on baseline day I. Scheduled sleep episodes in complete darkness (horizontal black bars). Subject 1839 (closed circle) was scheduled to the 24.0-hour day protocol (A). During the imposed 24.0-hour segment, melatonin onset appears stable and occurs near to lights out. Circadian period delayed during the 28-hour day length forced desynchrony protocol (days 36-47) indicating an intrinsic period longer than 24.0 -hours (detailed forced desynchrony data was reported previously). ${ }^{19,29}$ Subject I8J5 (closed triangle) was scheduled to the 24.6-hours day protocol (B). During the imposed 24.6-hour segment lights out and lights on are delayed by 36 minutes each day. Melatonin onset occurred near to lights out for the subject during baseline days, whereas during the 24.6 -hour segment, melatonin onset is progressively phase advanced relative to lights out. Circadian period advanced during the forced desynchrony protocol indicating an intrinsic period shorter than 24.0 hours. Data for the current analyses comes from days 6 to 23 of the protocol. Blood was collected on day 6 to assess 24 -hour baseline leptin levels and day 7 to assess circadian melatonin phase. The average day of leptin reassessment was day 22 and the average day of melatonin phase reassessment was day 23 of the protocol.

\section{Biological specimen analyses}

Samples were stored at $-80^{\circ} \mathrm{C}$ until assayed. Plasma melatonin levels were assayed using radioimmunoassay (RIA) ${ }^{125} \mathrm{I}$ (Elias USA, Inc., Osceola, WI) with a sensitivity of $2.5 \mathrm{pg} / \mathrm{mL}$ and an intra- and interassay coefficients of variation of $5.9 \%$ and $9.8 \%$, respectively. Plasma leptin levels were measured by human leptin RIA (Linco Research, St. Charles, MO), with a sensitivity of $0.5 \mathrm{ng} / \mathrm{mL}$ and an intra- and interassay coefficient of variation of $5.9 \%$ and $5.8 \%$, respectively.

The circadian phase of the dim light melatonin onset (DLMO25\%) was calculated using the linear interpolated point in time when melatonin levels reached $25 \%$ of the fitted trough to peak amplitude of the third harmonic of each subject's data from the constant routine. ${ }^{17,29}$

Blood collection was occasionally delayed from the scheduled time due to sampling difficulties (eg, $10 \mathrm{~min}$ late); therefore, plasma leptin data were linear interpolated between points and anchored to scheduled waketime to provide equidistant time points to be analyzed. Trapezoidal area under the curve (AUC) and average leptin levels during scheduled wakefulness and sleep were calculated.

\section{Sleep analyses}

Sleep recordings were obtained with a Vitaport Digital Recorder (Temec Instruments, Kerkrade, The Netherlands). High and low pass filters were set at 0.25 and $70 \mathrm{~Hz}$, respectively. Impedances were below $10 \mathrm{k} \Omega$. Sleep records were scored blind to experimental group from central EEG brain site $\mathrm{C} 3-\mathrm{A} 2$ or $\mathrm{C} 4-\mathrm{A} 1$ if $\mathrm{C} 3$ was not available. ${ }^{33}$ Sleep stage data were averaged across two consecutive nights that did not include blood sampling: baseline nights 3-4, approximately 3-4 nights prior to leptin assessment and approximately 3-4 nights after leptin assessment. Due to data loss for one night, one subject had only one usable sleep recording for the post leptin assessment sleep analysis. Regardless of time in bed ( 8.0 or 8.2 hours) the first eight hours of each recording was used in the data analysis. ${ }^{19}$ Sleep parameters have been previously reported in detail for this protocol with a larger 
sample size ${ }^{19}$ thus, sleep measures are provided for the current participants primarily for descriptive purposes.

\section{Statistical analyses}

Changes in dependent measures were analyzed with mixed model repeated measures ANOVA techniques. Unless noted, statistics describe results for interactions between groups (synchronized vs nonsynchronized) and time (day of study or time-of-day). Planned comparisons between baseline and day 22 were computed within each group and between groups.

\section{Results}

\section{Circadian entrainment}

As reported previously, ${ }^{19,29}$ some participants maintained circadian synchronization while others failed to synchronize to the scheduled 24.0- or 24.6-hour day lengths. Subjects were classified as not entrained because their DLMO25\% progressively drifted outside the normal range and the 95\% CI for their observed circadian period of their melatonin rhythm did not include the scheduled day length. ${ }^{19,29}$ Half of the current participants (six male, one female) were characterized as being "synchronized" and the remaining participants (six male, one female) were characterized as "nonsynchronized" in the previous reports. Levels of the sleep promoting hormone melatonin were high during scheduled sleep in the synchronized group and low during scheduled sleep in the nonsynchronized group. Figure 2 shows that melatonin onset was significantly earlier relative to scheduled sleep in the participants who failed to synchronize to the scheduled 24.0- or 24.6-hour day lengths [nonsynchronized group versus synchronized group (Day $\times$ group effect, $\left.\left.\mathrm{F}_{1,212}=7.81, P=0.02\right)\right]$. As reported previously, ${ }^{19,29}$ failure to adapt to the scheduled day length was dependent upon individual differences in intrinsic circadian period relative to the scheduled day length and light intensity.

\section{Sleep}

As reported previously ${ }^{19}$ sleep was more disrupted in the nonsynchronized group later in the protocol as the degree of circadian misalignment became greater. Table 1 shows that the latency to sleep, minutes of stage 2 sleep and total sleep time (TST) and sleep efficiency (SE) significantly decreased, whereas wakefulness after sleep onset (WASO) significantly increased in the nonsynchronized group for the sleep episodes the day after leptin assessment compared to baseline. Latency to sleep significantly increased in the synchronized group compared to baseline. The amount of stages $1,3 / 4$ and rapid eye movement (REM) sleep, as well
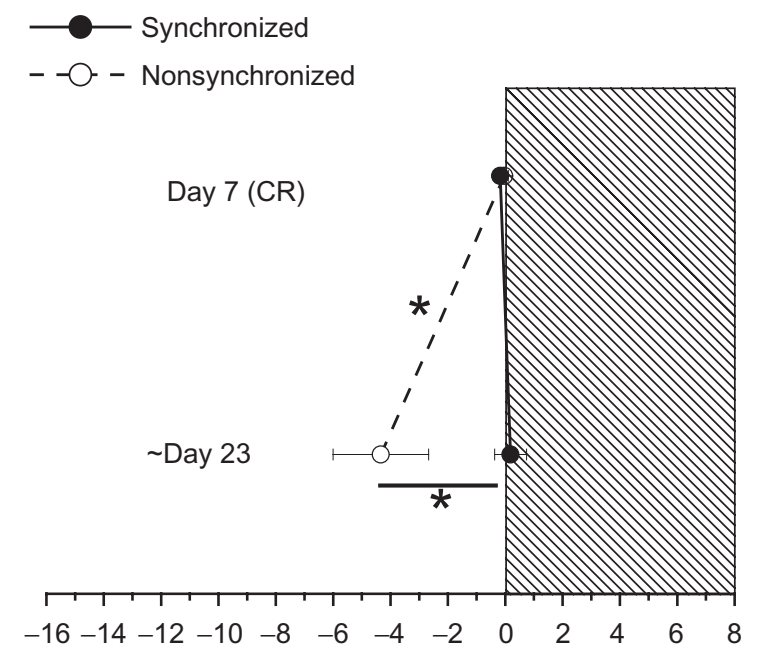

Hours from scheduled sleep

Figure 2 Circadian phase angle as assessed by the timing of the dim light melatonin onset (DLMO25\%) in plasma relative to scheduled sleep. Day 7 (baseline) and 23 represent average day of melatonin assessment during the inpatient protocol. On day 23 , the DLMO25\% occurred earlier in the nonsynchronized group compared to baseline and compared to the synchronized group. The earlier onset of melatonin in the nonsynchronized group resulted in higher melatonin levels during scheduled wakefulness and lower melatonin levels during scheduled sleep. Note that nonsynchronized group was synchronized at baseline. ${ }^{*} P<0.05$.

as the latency to REM sleep did not significantly change across the protocol for either group; although as reported previously, the larger degree of circadian misalignment observed later in the protocol for the nonsynchronized group resulted in a shorter REM latency. ${ }^{19}$

\section{Weight and caloric intake}

Table 2 shows that there were nonsignificant changes in weight and caloric intake for both groups across the protocol.

\section{Leptin}

There was a significant time of day effect $\left(\mathrm{F}_{23,276}=\right.$ 33.36, $P<0.0001)$ and a nonsignificant day $\times$ group interaction $\left(\mathrm{F}_{1,12}=3.25, P=0.097\right)$ for leptin levels. Figure 3 shows that leptin levels were lowest several hours after scheduled wakefulness, increased across the day and were highest during scheduled sleep regardless of group or day of study. However, leptin levels were significantly lower in the nonsynchronized group on day 22 compared to baseline, when sleep and wakefulness occurred out of phase with internal biological time; whereas leptin levels were significantly higher in the synchronized group on day 22 compared to baseline.

There was a significant wakefulness $\times$ sleep interaction $\left(\mathrm{F}_{1,12}=102.59, P<0.00001\right)$ and a nonsignificant day $\times$ group interaction $\left(\mathrm{F}_{1,12}=3.34, P=0.092\right)$ for AUC leptin levels. Figures $4 \mathrm{a}$ and $4 \mathrm{~b}$ show that AUC leptin levels were 
Table I Sleep parameters at baseline (average of nights 3 and 4), pre-leptin analysis (average of nights I7 and I8) and post-leptin analysis (average of nights 25 and 26 )

\begin{tabular}{|c|c|c|c|c|c|c|}
\hline & \multicolumn{6}{|l|}{ Sleep episode } \\
\hline & \multicolumn{2}{|l|}{ Baseline } & \multicolumn{2}{|c|}{ Pre-leptin analysis } & \multicolumn{2}{|c|}{ Post-leptin analysis } \\
\hline & Synchronized & Nonsynchronized & Synchronized & Nonsynchronized & Synchronized & Nonsynchronized \\
\hline Sleep latency (min) & $13.8 \pm 2.7$ & $13.6 \pm 2.2$ & $16.0 \pm 4.4$ & $6.3 \pm 3.1$ & $24.6 \pm 6.0^{\prime}$ & $4.4 \pm 0.6^{2,3}$ \\
\hline REM latency (min) & $69.5 \pm 3.5$ & $63.9 \pm 2.9$ & $84.8 \pm 19.8$ & $73.8 \pm 15.2$ & $75.8 \pm 6.6$ & $45.7 \pm 8.7$ \\
\hline Sleep efficiency (\%) & $88.0 \pm 4.6$ & $91.0 \pm 1.5$ & $89.0 \pm 3.6$ & $89.0 \pm 3.9$ & $91.0 \pm 1.9$ & $83.0 \pm 3.8^{1}$ \\
\hline \multicolumn{7}{|c|}{ Amount of sleep stages (min) } \\
\hline WASO & $45.9 \pm 21.5$ & $25.8 \pm 8.6$ & $33.3 \pm 16.1$ & $37.0 \pm 19.1$ & $17.8 \pm 7.1$ & $74.7 \pm 18.6^{1}$ \\
\hline Stage I & $35.5 \pm 5.3$ & $46.3 \pm 4.3$ & $34.5 \pm 3.4$ & $42.2 \pm 5.8$ & $40.0 \pm 5.8$ & $50.0 \pm 6.9$ \\
\hline Stage 2 & $217.9 \pm 18.7$ & $235.2 \pm 11.5$ & $217.0 \pm 15.1$ & $226.3 \pm 19.5$ & $219.6 \pm 9.9$ & $200.6 \pm 12.3^{1}$ \\
\hline Stage $3+4$ & $60.4 \pm 8.4$ & $44.7 \pm 9.6$ & $63.6 \pm 5.3$ & $36.8 \pm 9.5$ & $58.7 \pm 7.7$ & $43.2 \pm 10.1$ \\
\hline REM sleep & $106.2 \pm 10.0$ & $\mid 12.1 \pm 5.8$ & $112.6 \pm 11.2$ & $120.8 \pm 7.0$ & $119.3 \pm 9.1$ & $104.4 \pm 7.8$ \\
\hline Total sleep time & $420.0 \pm 22.2$ & $438.4 \pm 7.2$ & $427.8 \pm 17.4$ & $426.1 \pm 18.6$ & $437.5 \pm 9.1$ & $398.1 \pm 17.4^{\prime}$ \\
\hline
\end{tabular}

Notes: Results are reported as mean \pm SEM. 'P $<0.05$ with respect to baseline, ${ }^{2} P=0.052$ with respect to baseline, ${ }^{3} P<0.05$ with respect to group.

significantly reduced in the nonsynchronized group during scheduled wakefulness on day 22 compared to baseline. No significant changes in AUC leptin levels were observed in the synchronized group during scheduled wakefulness, nor were there any significant changes in AUC leptin levels in either group during scheduled sleep.

There was a significant wakefulness $\mathrm{x}$ sleep interaction $\left(\mathrm{F}_{1,12}=44.528, P<0.0001\right)$ and a nonsignificant day $\times$ group interaction $\left(\mathrm{F}_{1,12}=3.40, P=0.090\right)$ for average leptin levels. Figures $4 \mathrm{c}$ and $4 \mathrm{~d}$ show that average leptin levels were significantly reduced in the nonsynchronized group during scheduled wakefulness by $10 \%$ and there was a nonsignificant reduction in leptin levels during sleep on day 22 compared to baseline. No significant changes in average leptin levels were observed in the synchronized group during scheduled wakefulness; however, a significant increase in average leptin levels was observed in the synchronized group during scheduled sleep.

\section{Discussion}

Leptin levels were reduced in participants who did not maintain a normal relationship between the imposed sleep-wakefulness schedule and internal biological time. The degree of circadian misalignment (ie, $\sim 4$-hour change from baseline) resulted in significantly lower leptin levels, especially during scheduled wakefulness. Specifically, the AUC and average leptin levels were significantly reduced by $10 \%$ during scheduled wakefulness in the misaligned group. The $10 \%$ decrease in leptin levels during scheduled wakefulness is nearly half of the $22 \%$ decrease in leptin caused by three days of food restriction to $\sim 70 \%$ of energy requirements; the latter is a potent stimulus for increasing food intake. Thus, the change in leptin levels we observed are likely to be physiologically meaningful. The shape of the 24-hour leptin profile was not altered by weeks of circadian misalignment suggesting that sleep-wakefulness state and associated meal intake have a larger impact than circadian phase on the daily leptin profile. Leptin levels were always lowest several hours after scheduled wakefulness, increased across the day and were highest during scheduled sleep for synchronized and nonsynchronized groups.

Schoeller and colleagues ${ }^{2}$ reported no change in 24-hour leptin levels during four days of simulated jet lag. However, their finding during acute circadian misalignment maybe related to low statistical power $(\mathrm{N}=4)$. Scheer and

Table 2 Average weight and caloric intake for the day of and four days prior to baseline leptin assessment and leptin reassessment respectively

\begin{tabular}{llllll}
\hline & \multicolumn{2}{l}{ Synchronized } & & \multicolumn{2}{l}{ Nonsynchronized } \\
\cline { 2 - 3 } & Baseline leptin assessment & Leptin reassessment & & Baseline leptin assessment & Leptin reassessment \\
\hline Weight $(\mathrm{kg})$ & $79.2 \pm 5.2$ & $78.6 \pm 5.2$ & $71.9 \pm 4.2$ & $71.2 \pm 4.5^{1}$ \\
Caloric Intake $(\mathrm{kcal})^{2}$ & $2445.3 \pm 197.7$ & $2311.1 \pm 197.6^{3}$ & & $2215.9 \pm 214.2$ & $2132.8 \pm 275.2$ \\
\hline
\end{tabular}

Notes: ${ }^{\prime} P=0.09$ with respect to baseline; ${ }^{2} n=5$ for caloric intake per group; ${ }^{3} P=0.07$ with respect to baseline. 
A

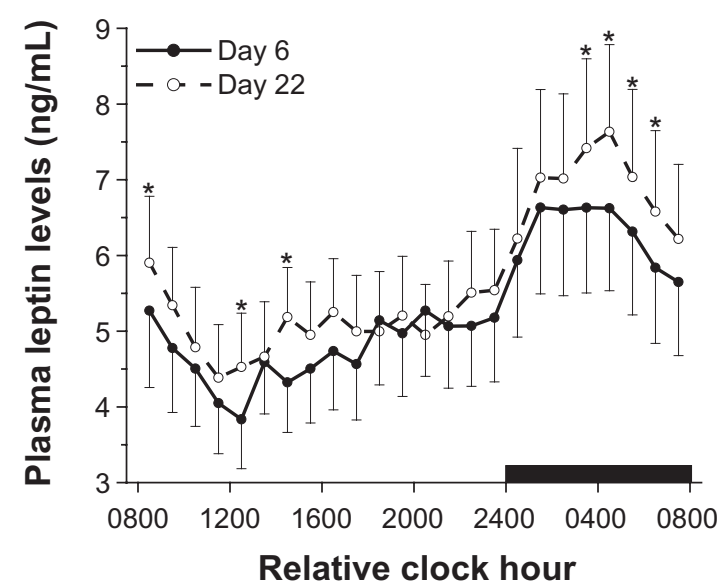

B

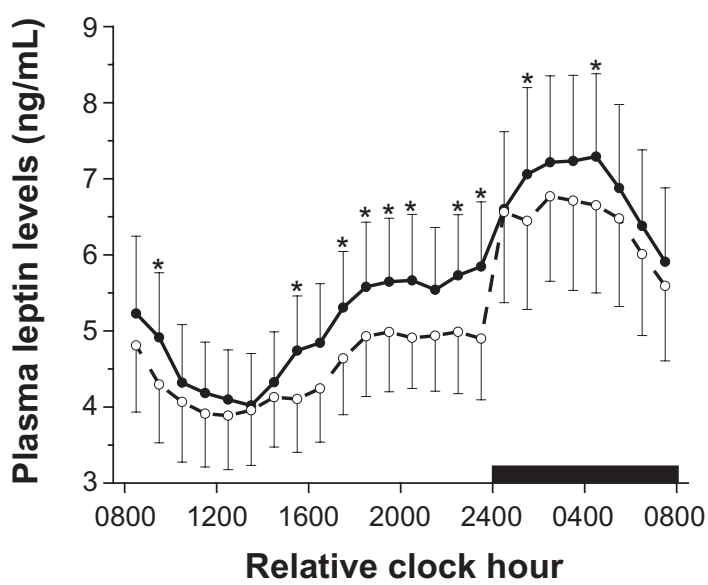

Figure 3 Mean $( \pm S E M)$ 24-hour profiles of leptin in the synchronized group (A) and nonsynchronized group (B). Black bar represents schedule sleep episode. Note that nonsynchronized group was synchronized at baseline.

Notes: ${ }^{*} P<0.05$ compared to baseline.

colleagues $^{8}$ reported significant reductions in leptin levels using an experimental paradigm that induced rapid changes in environmental time equivalent to flying four time zones westward each day. Specifically, they found leptin to be lower and sleep to be impaired when sleep was scheduled during the biological day. Findings of reduced leptin levels during circadian misalignment from the current study are consistent with and extend the findings by Scheer and colleagues. ${ }^{8}$ In the
A

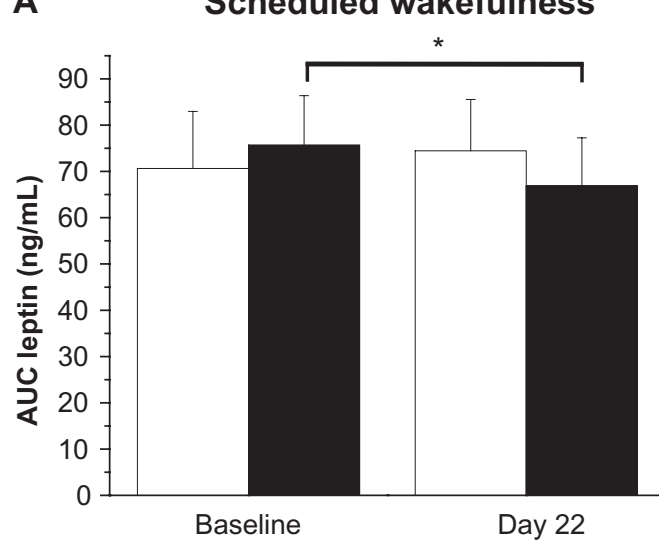

C

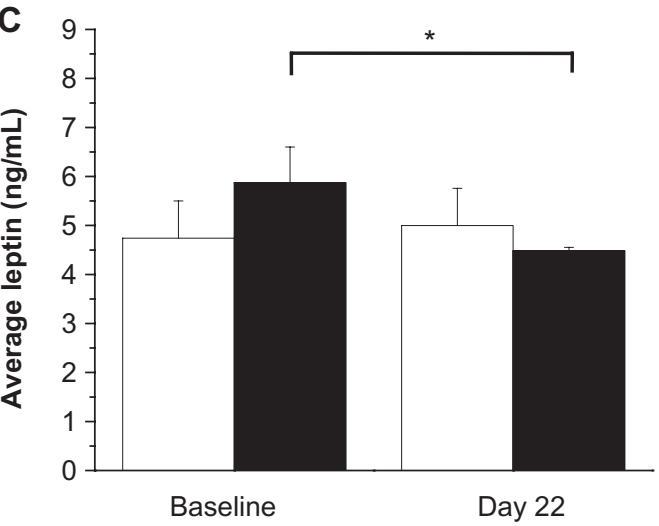

B

\section{Scheduled sleep}
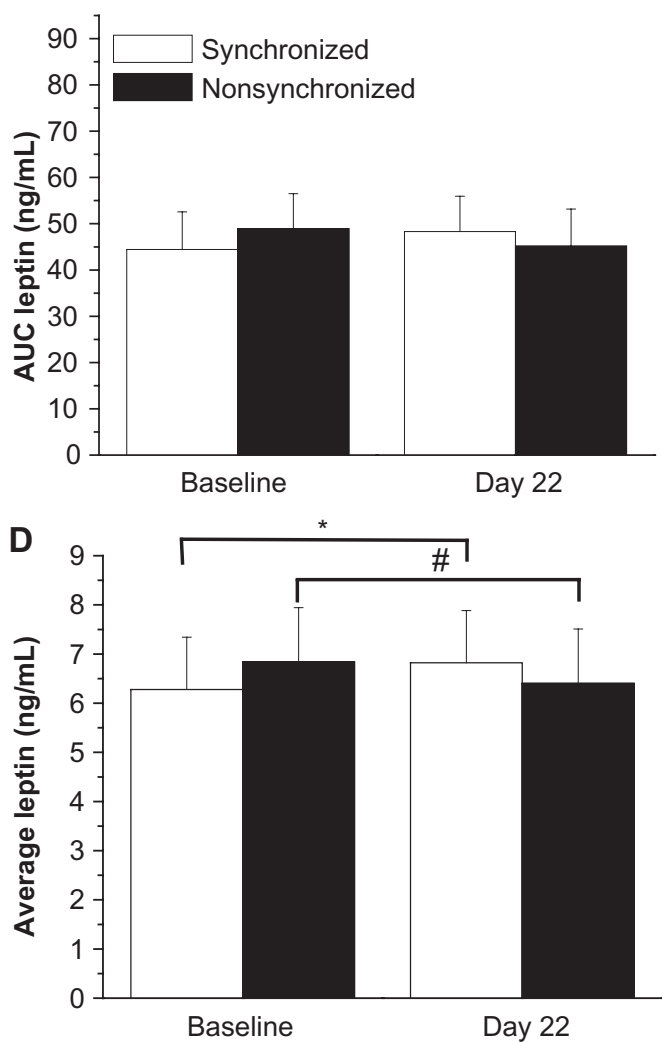

Figure 4 Integrated area under the curve leptin levels during scheduled wakefulness (A) and scheduled sleep (B). Day 22 denotes the average day of study when leptin was reassessed. Average leptin levels during scheduled wakefulness (C) and scheduled sleep (D).

Notes: The nonsynchronized group was synchronized at baseline. ${ }^{* P}<0.05$ compared to baseline. ${ }^{\# P}=0.07$ compared to baseline. 
current study, the duration of circadian misalignment was longer and the daily change in the degree of misalignment between the sleep and wakefulness schedule and internal biological time was slower, and the on average $\sim 4$-hour change in phase angle from baseline was smaller than the largest phase angle difference examined in the study by Scheer and colleagues. ${ }^{8}$ Future studies could assess leptin levels on a weekly basis to determine if changes in leptin are observed at other phase angle differences between sleep timing and circadian phase when circadian misalignment occurs slowly as compared to rapid large shifts in sleep timing relative to circadian phase. The pattern of circadian misalignment observed in the current study ${ }^{19,29}$ is consistent with that seen in non-24-hour disorder (free running type). Non-24-hour sleep-wakefulness disorder occurs in sighted and blind individual $\mathrm{s}^{34}$ whose circadian clocks fail to entrain to the 24-hour day. Failure to entrain to the imposed day length also occurs under altered work operations such as during space missions and submarine operations where crewmembers are often required to live on non-24-hour day lengths. ${ }^{18,19,22,29,32,35}$ The significant reduction in leptin levels observed during weeks of circadian misalignment suggests that these populations may be at greater risk of metabolic disorders associated with lower leptin levels.

The significant reduction in leptin levels observed during weeks of circadian misalignment in the current study was not associated with significant changes in the amounts of Stages 1, 3/4, REM sleep, or differences in total sleep duration between groups. However, during circadian misalignment, sleep onset latency, stage 2 sleep time, and total sleep time were shorter and wakefulness after sleep onset was greater in the nonsynchronized group compared to baseline. At baseline, participants in the nonsynchronized group slept on average 7.3 hours; whereas on the days prior to and after the day of leptin reassessment, participants in the nonsynchronized group slept on average 7.1 and 6.6 hours, respectively. The reduction in total sleep time for the nonsynchronized group is much smaller than the amount of sleep restriction reported to reduce leptin levels in prior studies. Specifically, Spiegel and colleagues ${ }^{12}$ reported significantly reduced 24-hour leptin levels following six nights of sleep restriction, averaging 3.8 hours sleep per night versus seven days sleep extension, averaging 8.9 hours of sleep per night. Furthermore, Spiegel and colleagues ${ }^{11}$ reported that two nights sleep extension averaging 9.1 hours of sleep per night compared to two nights of sleep restriction averaging 3.9 hours of sleep per night significantly increased leptin levels. Scheer and colleagues ${ }^{8}$ reported a reduction in sleep efficiency from $84 \%$ to $67 \%$ during acute rapid circadian misalignment and that significant reductions in leptin were observed even after statistically correcting for sleep duration the day prior. Our finding that leptin levels were significantly lower in the nonsynchronized group after obtaining $\sim 6.6$ to 7.1 hours of sleep per night suggests that changes in leptin levels may respond to smaller amounts of sleep loss than previously recognized. It is also likely that scheduled wakefulness during the biological night in the nonsynchronized group contributed to the observed reduction in leptin levels. In practice, it is impossible to separate the influence of circadian misalignment and sleep loss on leptin levels, as sleep loss occurs as a result of circadian misalignment. In fact, the sleep and circadian systems interact to modulate physiology and changes in one system, often influences the other. For example, sleep restriction is reported to alter circadian timing ${ }^{36}$ and thus, an altered relationship between circadian phase and sleep may contribute to prior findings of reduced leptin during sleep restriction. Mechanistically, changes in peripheral clock gene expression may be associated with the changes in leptin found in our study. Circadian clock genes are reported to be expressed rhythmically in adipose tissue, which is the primary source of circulating leptin levels, and feeding restricted to a specific time of day is reported to induce circadian phase shifts in these clock genes. ${ }^{37}$ In addition, mutations of circadian clock genes have been reported to be associated with obesity and high leptin levels, ${ }^{38}$ similar to findings of an association between obesity and leptin resistance in humans. ${ }^{1}$

In the synchronized group there was a significant increase in leptin levels across several time points during scheduled wakefulness and sleep. Furthermore, there was a significant increase in average leptin levels during sleep. It is possible that the small nonsignificant $\sim 18 \mathrm{~min}$ increase in total sleep time from baseline may have contributed to the higher leptin levels in this group. Such an interpretation is consistent with prior sleep extension findings, ${ }^{11,12}$ but would indicate that a small increase in total sleep time can influence energy balance mediators in humans.

The shape of the 24-hour leptin profile was similar for synchronized and nonsynchronized groups. Plasma leptin levels were lowest several hours after scheduled wakefulness, increased across the day and were highest during scheduled sleep. The observed diurnal variation in 24-hour leptin levels is consistent with previous findings. ${ }^{2,4-10}$ Our finding that the phase relationship between circadian phase and scheduled sleep and wakefulness was on average earlier for participants in the synchronized group, but that the diurnal profile of the 
24-hour leptin levels were similar for the synchronized and nonsynchronized group, indicates that the 24-hour leptin profile is more strongly regulated by the wakefulness-sleep cycle than by internal circadian timing. The primary factor(s) of the wakefulness-sleep schedule that influence the shape of the diurnal profile of 24-hour leptin levels are unknown, but may include an increase in leptin levels due to sleep, ${ }^{5,12,39}$ changes in metabolic function during sleep and wakefulness ${ }^{8,40,41}$ and/or changes in response to food intake across wakefulness and fasting during sleep. ${ }^{2,42,43}$ It is possible that the reduction in leptin levels we observed during circadian misalignment may in part be related to participants eating for weeks at an internal biological time when the master circadian clock is promoting sleep and when metabolic responsiveness to food intake is altered. ${ }^{42,44,45}$

Lower leptin levels during wakefulness associated with circadian misalignment may alter the energy balance. It has been previously reported that reduced leptin levels during sleep restriction are associated with increased hunger ratings. ${ }^{11}$ In the current study, we did not assess subjective hunger or energy expenditure. Furthermore, the timing of meals was scheduled and the caloric content of meals was strictly controlled to maintain subject's weight. Because weight was maintained in the current subjects, it is possible that the lower leptin levels during circadian misalignment represent a change in fat mass. Future studies should examine changes in fat and fat free mass, energy balance, and ad libitum feeding behavior during weeks of circadian misalignment.

The current study was designed as a ground based model of exploration class space missions, including exposure to the Martian Sol. ${ }^{19,29}$ Most individuals in the circadian misalignment group were exposed to the 24.6-hour day, an approximation of the day length of the planet Mars. That circadian misalignment resulted in altered leptin levels suggests that effective countermeasures are needed to adapt the circadian system to the Martian day ${ }^{32,35}$ to prevent alterations in leptin levels and perhaps altered metabolic physiology.

In summary, findings from the current study provide further evidence that changes in the sleep and circadian systems influence the feeding system in humans. Circadian misalignment and associated sleep loss significantly reduced leptin levels during wakefulness, which may promote increased food intake under ad libitum intake conditions. Future research is needed to determine if food intake is increased in response to reduced leptin levels during circadian misalignment and whether such changes are associated with the increased risk of obesity and other metabolic disorders in populations where circadian misalignment is common, such as in shift workers and in patients with circadian sleep disorders.

\section{Acknowledgments}

We would like to thank Charles A Czeisler, PhD, MD, and the GCRC dieticians and technicians for study support. This study was funded by NIH HL073196, by NASA Cooperative Agreement NCC9-58 with the National Space Biomedical Research Institute and NASA, and by General Clinical Research Center Grants GCRC M01-RR02635 and M01-RR000051 from the National Center for Research Resources. The funding sources had no role in the design and conduct of the study; collection, management, analysis, and interpretation of the data and preparation, review, or approval of the manuscript. Dr Wright received consulting fees, speaker's fees, clinical trial research contracts and investigator-initiated research grants from Cephalon, Inc. Additionally he has received consulting fees, speakers fees, investigator-initiated research grants and education grants from Takeda Pharmaceuticals North America. Dr Wright has also received consulting fees from Novartis; serving on the advisory board for Zeo Inc. (formerly Axon Labs, Inc) and receiving Nonqualified Stock Options.

\section{References}

1. Arch JR. Central regulation of energy balance: inputs, outputs and leptin resistance. Proc Nutr Soc. 2005;64(1):39-46.

2. Schoeller DA, Cella LK, Sinha MK, Caro JF. Entrainment of the diurnal rhythm of plasma leptin to meal timing. J Clin Invest. 1997;100(7):1882-1887.

3. Sinha MK, Opentanova I, Ohannesian JP, Kolaczynski JW, Heiman ML, Hale J, et al. Evidence of free and bound leptin in human circulation. Studies in lean and obese subjects and during short-term fasting. J Clin Invest. 1996;98(6):1277-1282.

4. Saad MF, Riad-Gabriel MG, Khan A, et al. Diurnal and ultradian rhythmicity of plasma leptin: effects of gender and adiposity. J Clin Endocrinol Metab. 1998;83(2):453-459.

5. Simon C, Gronfier C, Schlienger JL, Brandenberger G. Circadian and ultradian variations of leptin in normal man under continuous enteral nutrition: relationship to sleep and body temperature. J Clin Endocrinol Metab. 1998;83(6):1893-1899.

6. Mullington JM, Chan JL, Van Dongen HP, et al. Sleep loss reduces diurnal rhythm amplitude of leptin in healthy men. J Neuroendocrinol. 2003;15(9):851-854.

7. Langendonk JG, Pijl H, Toornvliet AC, et al. Circadian rhythm of plasma leptin levels in upper and lower body obese women: influence of body fat distribution and weight loss. J Clin Endocrinol Metab. 1998;83(5):1706-1712.

8. Scheer FA, Hilton MF, Mantzoros CS, Shea SA. Adverse metabolic and cardiovascular consequences of circadian misalignment. Proc Natl Acad Sci US A. 2009;106(11):4453-4458.

9. Chin-Chance C, Polonsky KS, Schoeller DA. Twenty-four-hour leptin levels respond to cumulative short-term energy imbalance and predict subsequent intake. J Clin Endocrinol Metab. 2000;85(8):2685-2691. 
10. Elimam A, Marcus C. Meal timing, fasting and glucocorticoids interplay in serum leptin concentrations and diurnal profile. Eur J Endocrinol. 2002;147(2):181-188.

11. Spiegel K, Tasali E, Penev P, Van Cauter E. Brief communication: Sleep curtailment in healthy young men is associated with decreased leptin levels, elevated ghrelin levels, and increased hunger and appetite. Ann Intern Med. 2004;141(11):846-850.

12. Spiegel K, Leproult R, L'Hermite-Baleriaux M, Copinschi G, Penev PD, Van Cauter E. Leptin levels are dependent on sleep duration: Relationships with sympathovagal balance, carbohydrate regulation, cortisol, and thyrotropin. J Clin Endocrinol Metab. 2004;89(11):5762-5771.

13. Shea SA, Hilton MF, Orlova C, Ayers RT, Mantzoros CS. Independent circadian and sleep/wake regulation of adipokines and glucose in humans. J Clin Endocrinol Metab. 2005;90(5):2537-2544.

14. Chan JL, Bullen J, Lee JH, Yiannakouris N, Mantzoros CS. Ghrelin levels are not regulated by recombinant leptin administration and/or three days of fasting in healthy subjects. J Clin Endocrinol Metab. 2004;89(1):335-343.

15. Taheri S, Lin L, Austin D, Young T, Mignot E. Short sleep duration is associated with reduced leptin, elevated ghrelin, and increased body mass index. PLoS Med. 2004;1(3):1-8.

16. Gillette MU, Abbott SM. Biological timekeeping. Sleep Med Clin. 2009;4(2):99-110

17. Wright KP Jr, Gronfier C, Duffy JE, Czeisler CA. Intrinsic period and light intensity determine the phase relationship between melatonin and sleep in humans. J Biol Rhythms. 2005;20(2):168-177.

18. Dijk DJ, Neri DF, Wyatt JK, Ronda JM, Riel E, Ritz-De Cecco A, et al. Sleep, performance, circadian rhythms, and light-dark cycles during two space shuttle missions. Am J Physiol. 2001;281(5):R1647-R1664.

19. Wright KP Jr, Hull JT, Hughes RJ, Ronda JM, Czeisler CA. Sleep and wakefulness out of phase with internal biological time impairs learning in humans. J Cogn Neurosci. 2006;18(4):508-521.

20. Åkerstedt TA, Wright KP Jr. Sleep loss and fatigue in shift work and shift work disorder. Sleep Med Clin. 2009;4(2):257-271.

21. Eastman CI, Burgess HJ. How to travel the world without jet lag. Sleep Med Clin. 2009;4(2):241-255.

22. Kelly TL, Neri DF, Grill JT, et al. Nonentrained circadian rhythms of melatonin in submariners scheduled to an 18-hour day. J Biol Rhythms. 1999;14(3):190-196.

23. Basics of circadian biology and circadian rhythm sleep disorders. Sleep Med Clin. 2009;4(2):99-312.

24. Wirz-Justice A, Bromundt V, Cajochen C. Circadian disruption and psychiatric disorders: the importance of entrainment. Sleep Med Clin. 2009;4(2):273-284.

25. Laposky AD, Turek FW. Physiologic and health consequences of circadian disruption (in animal models). Sleep Med Clin. 2009;4(2):127-142.

26. Litinski M, Scheer FAJL, Shea SA. Influence of the circadian system on disease severity. Sleep Med Clin. 2009;4(2):143-163.

27. Bartness TJ, Song CK, Demas GE. SCN efferents to peripheral tissues: implications for biological rhythms. J Biol Rhythms. 2001;16(3):196-204.

28. Ishizaki M, MorikawaY, Nakagawa H, Honda R, Kawakami N, Haratani T, et al. The influence of work characteristics on body mass index and waist to hip ratio in Japanese employees. Ind Health. 2004;42(1):41-49.
29. Wright KP Jr, Hughes RJ, Kronauer RE, Dijk DJ, Czeisler CA. Intrinsic near-24-h pacemaker period determines limits of circadian entrainment to a weak synchronizer in humans. Proc Natl Acad Sci U S A. 2001;98(24):14027-14032.

30. Duffy JF, Wright KP Jr. Entrainment of the human circadian system by light. J Biol Rhy. 2005;28(4):326-338.

31. Czeisler CA, Wright KP Jr. Influence of light on circadian rhythmicity in humans. In: Turek FW, Zee PC, editors. Regulation of Sleep and Circadian Rhythms. New York. NY: Marcel Dekker, Inc.; 1999. p. 149-180.

32. Gronfier C, Wright KP Jr, Kronauer RE, Czeisler CA. Entrainment of the human circadian pacemaker to longer-than-24-h days. Proc Natl Acad Sci U S A. 2007;104(21):9081-9086.

33. Rechtschaffen A, Kales A. A Manual of Standardized Terminology, Techniques and Scoring System for Sleep Stages of Human Subjects. Washington, DC: US Government Printing Office; 1968.

34. Uchiyama M, Lockley SW. Non-24-hour sleep-wake syndrome in sighted and blind patients. Sleep Med Clin. 2009;4(2):195-211.

35. Scheer FA, Wright KP Jr, Kronauer RE, Czeisler CA. Plasticity of the intrinsic period of the human circadian timing system. PLoS One. 2007;2(1):e721.

36. Burgess HJ, Eastman CI. Early versus late bedtimes phase shift the human dim light melatonin rhythm despite a fixed morning lights on time. Neurosci Lett. 2004;356(2):115-118.

37. Zvonic S, Ptitsyn AA, Conrad SA, Scott LK, Floyd ZE, Kilroy G, et al. Characterization of peripheral circadian clocks in adipose tissues. Diabetes. 2006;55(4):962-970.

38. Turek FW, Joshu C, Kohsaka A, et al. Obesity and metabolic syndrome in circadian Clock mutant mice. Science. 2005;308(5724):1043-1045.

39. Laposky AD, Shelton J, Bass J, Dugovic C, Perrino N, Turek FW. Altered sleep regulation in leptin-deficient mice. Am J Physiol Regul Integr Comp Physiol. 2006;290(4):R894-R903.

40. Sinton CM, Fitch TE, Gershenfeld HK. The effects of leptin on REM sleep and slow wave delta in rats are reversed by food deprivation. J Sleep Res. 1999;8(3):197-203.

41. Hilton LK, Loucks AB. Low energy availability, not exercise stress, suppresses the diurnal rhythm of leptin in healthy young women. Am J Physiol Endocrinol Metab. 2000;278(1):E43-E49.

42. Wellhoener P, Fruehwald-Schultes B, Kern W, et al. Glucose metabolism rather than insulin is a main determinant of leptin secretion in humans. J Clin Endocrinol Metab. 2000;85(3):1267-1271.

43. Sonnenberg GE, Krakower GR, Hoffmann RG, Maas DL, Hennes MM, Kissebah AH. Plasma leptin concentrations during extended fasting and graded glucose infusions: relationships with changes in glucose, insulin, and FFA. J Clin Endocrinol Metab. 2001;86(10):4895-4900.

44. Aparicio NJ, Puchulu FE, Gagliardino JJ, et al. Circadian variation of the blood glucose, plasma insulin and human growth hormone levels in response to an oral glucose load in normal subjects. Diabetes. 1974;23(2):132-137.

45. Van Cauter E, Polonsky KS, Scheen AJ. Roles of circadian rhythmicity and sleep in human glucose regulation. Endocr Rev. 1997;18(5):716-738.
Nature and Science of Sleep

\section{Publish your work in this journal}

Nature and Science of Sleep is an international, peer-reviewed, open access journal covering all aspects of sleep science and sleep medicine, including the neurophysiology and functions of sleep, the genetics of sleep, sleep and society, biological rhythms, dreaming, sleep disorders and therapy, and strategies to optimize healthy sleep. The journal welcomes

\section{Dovepress}

original research, clinical \& epidemiological studies, reviews \& evaluations, case reports and extended reports. The manuscript management system is completely online and includes a very quick and fair peerreview system, which is all easy to use. Visit http://www.dovepress.com/ testimonials.php to read real quotes from published authors. 